23

\title{
Высокостабильный источник спектральных линий
}

\author{
() В.К. Битюков ${ }^{1}$, Р.А. Горбунов ${ }^{2}$, Д.С. Симачков ${ }^{1, \uparrow, ~ А . В . ~ Ф р у н з е ~}{ }^{1,3}$ \\ ${ }^{1}$ МИРЭА - Российский технологический университет, \\ 119454 Москва, Россия \\ 2 „Ростест-Москва“, \\ 117418 Москва, Россия \\ ${ }^{3}$ ННТП „Термоконт“, \\ 119361 Москва, Россия \\ "e-mail: simachkov@mirea.ru \\ Поступила в редакцию 19.02.2018 г. \\ В окончательной редакции 11.12.2018 г. \\ Принята к публикации 18.12.2018 г.
}

В спектроскопии предъявляются повышенные требования к параметрам излучения ламп с полым катодом. Для поверки спектрометрического оборудования необходимо создание высокостабильного источника спектральных линий, интенсивность которого на заданных спектральных линиях будет сохраняться неизменной $(\leq 1 \%)$ в течение длительного времени. Предложено решение этой задачи и представлена схема экспериментального стенда для лампы с полым катодом с контуром оптико-электронной отрицательной обратной связи. Сигнал обратной связи управляет источником тока, являющимся функциональным узлом специализированного блока электропитания и формирующим электрический ток, протекающий через лампу. Представлены результаты экспериментального исследования с соответствующим метрологическим анализом. Предложенное техническое решение построения стабильного источника спектральных линий позволило получить высокий уровень стабильности спектральных линий как в кратковремен.ном, так и в долговременном интервале.

DOI: $10.21883 / \mathrm{OS} .2019 .04 .47525 .50-18$

\section{Актуальность создания высокостабильных источников спектральных линий}

Одним из наиболее распространенных источников спектральных линий являются лампы с полым катодом (ЛСП) [1]. Они появились в 50-х годах ХХ столетия и используются преимущественно в атомно-абсорбционном химическом анализе в качестве источников излучения на линиях поглощения анализируемых материалов [2].

Спектр излучения таких ламп определяется материалом катода и инертным газом-наполнителем. Энергия ионизации относительно низка, поэтому возбуждаются только спектральные линии, соответствующие переходам между достаточно близкими друг к другу энергетическими уровнями. Этим обусловлено то, что основные излучаемые линии ЛСП лежат в ультрафиолетовом диапазоне. Однако встречаются также и линии в видимом диапазоне, а в редких случаях - даже в ближней ИК области спектра.

Поскольку в нормальном состоянии ЛСП является вакуумным прибором, состав материала катода и газанаполнителя в котором в течение срока службы остается неизменным, то спектральный состав излучения каждой конкретной ЛСП также остается неизменным в ходе ее эксплуатации. В то же время интенсивность ее излучения изменяется даже при стабилизации разряд- ного тока через ЛСП. Для источников излучения в рутинном атомно-эмиссионном анализе это не является существенным недостатком, ограничивающим их применимость. Однако есть ряд направлений, где упомянутая нестабильность не позволяет использовать ЛСП.

В последнее время на стыке медицины и спектрального анализа возникло новое направление исследований, получившее название адресной доставки лекарств. Суть исследуемых методов состоит в том, чтобы подобрать спектр и мощность излучения, поступающего на кожу пациента, при которых с высокой долей вероятности гарантируется доставка лекарств к требуемому органу. Подобные методы сейчас находятся в стадии активных экспериментальных исследований. Однако общие требования к инструментарию и оборудованию, используемому в медицине, предусматривают нормирование и поверку их основных параметров. В случае использования в качестве оборудования для адресной доставки лекарств спектральных ламп, это означает нормирование и поверку не только длин волн излучения, но и их интенсивности.

Ведутся также и иные исследования по оценке влияния на человеческий организм светового воздействия с различным спектром излучения [3].

В качестве источников излучения чаще всего используют ЛСП. А для их поверки нужны эталонные источники, стабильность излучения которых значительно выше, чем у поверяемых ламп. Сейчас подобные высокостабильные эталонные источники спектральных линий 
отсутствуют. Да и требования к стабильности ламп, обеспечивающих доставку лекарств внутрь организма, еще не исследованы, возможно, с течением времени эти требования станут жестче, и обычные, типовые ЛСП, перестанут им удовлетворять.

Другим направлением применения высокостабильных источников спектральных линий является использование их в качестве излучателей в атомно-абсорбционных спектрометрах высокой точности, в том числе двустадийной атомизации [4]. Подобные спектрометры характеризуются самыми низкими пределами обнаружения элементов в химическом анализе. Во многом уровень предела обнаружения определяется временной стабильностью источника излучения. Если за время между калибровкой спектрометра и анализом пробы интенсивность излучения изменяется, то концентрация, соответствующая этому изменению, на калибровочной кривой прибора определяет минимум, ниже которого прибор может не почувствовать наличия элемента в пробе. Характерное время между калибровкой и измерением часто составляет несколько (до 10) минут. Поэтому для спектрометров максимальной чувствительности необходимо, чтобы за время 5-10 $\mathrm{min}$ интенсивность излучения лампы изменялась бы не более чем на $0.1 \%$. Выпускаемые серийно лампы этому требованию не удовлетворяют.

Еще одной областью применения высокостабильного источника спектральных линий является его использование для калибровки линеек ПЗС современных спектрометров. Сигнал, формируемый элементом ячейки ПЗС, помимо интенсивности засвечивающего ячейку светового сигнала, определяется большим числом факторов напряжением питания, температурой, шумами линейки. Пользователя же интересует стабильность сигналов, формируемых элементами ячеек линейки, при неизменном уровне ее засветки. Для этой цели также необходим прецизионный источник, формирующий спектральные линии, интенсивность которых изменяется на доли процента в течение длительного промежутка времени (месяц, год). Стандартная лампа с полым катодом этим требованиям не удовлетворяет.

Все вышесказанное подтверждает необходимость создания высоко-стабиль.ного источника спектральных линий, интенсивность излучения которого на заданных спектральных линиях будет сохраняться неизменной (на уровне $1 \%$ и менее) как кратковременно, так и в течение длительного времени.

\section{Схема и конструкция установки}

Как было отмечено выше, даже стабилизация тока через ЛСП не в состоянии обеспечить требуемой стабильности интенсивности излучения. Поэтому для решения поставленной задачи необходимо в контур управления ЛСП ввести отрицательную обратную связь

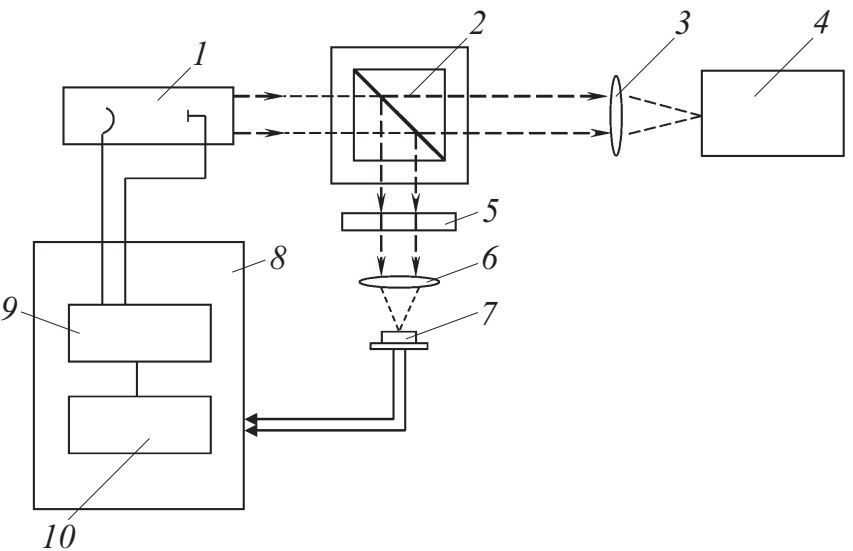

Схема высокостабильного источника спектральных линий.

(ООС), сигнал которой будет формироваться из выходного светового сигнала ЛСП фотодиодным датчиком с усилителем-преобразователем. Тогда любое изменение интенсивности выходного сигнала ЛСП будет приводить к изменению силы тока на выходе фотодиодного датчика, и как следствие, подачей в контур управления током ЛСП соответствующего напряжения, которое в конечном итоге возвращает интенсивность выходного сигнала ЛСП на прежний уровень. Схема ЛСП с упомянутым контуром ООС приведена на рисунке $[5,6]$.

Свет от спектральной лампы с полым катодом 1 попадает на светоделительный кубик 2, где разделяется на два пучка. Один пучок фокусируется линзой 3 и направляется в пользовательское устройство 4, которым является монохроматор или спектрометр. Другой пучок проходит через светофильтр 5, также фокусируется линзой 6 и попадает на фотодиод 7. Сигнал с фотодиода поступает в блок электропитания и управления лампы 8 , в котором находятся узел управления питанием лампы 9 и схема сравнения контура ООС 10. Если фотодиод регистрирует уменьшение интенсивности свечения лампы, т. е. напряжение фотодиода $U_{\text {фд }}$ меньше $U_{\text {опорное, то }}$ сигнал с выхода ООС увеличивает ток через лампу, и наоборот, при увеличении интенсивности свечения ток через лампу будет уменьшен.

\section{Исследование стабильности интенсивности спектральных линий}

Для экспериментального исследования использовался прецизионный цифровой мультиметр Щ-300, позволяющий измерять токи вплоть до $1 \cdot 10^{-10} \mathrm{~A}$.

В устройстве 4 (рисунок) был размещен дополнительный фотодиод, перед которым установлен узкополосный интерференционный светофильтр. Центр полосы пропускания светофильтра выбран совпадающим с длиной волны контролируемой спектральной линии (в данном случае это линия $\mathrm{Ne} 585 \mathrm{~nm}$ ), ширина полосы пропускания светофильтра по уровню 0.5 - около $10 \mathrm{~nm}$. Вблизи 
Таблица 1. Результаты измерений от 13.04.2017 г.

\begin{tabular}{|c|c|c|c|c|c|c|}
\hline \multirow{2}{*}{$\begin{array}{c}\text { Время, } \\
\text { h:min }\end{array}$} & \multicolumn{5}{|c|}{ Величина тока фотодиода $I_{p d}, \mathrm{nA}$} & \multirow{2}{*}{$\begin{array}{c}\text { Среднее } \\
\text { значение } \\
\text { с СКО }\end{array}$} \\
\hline & 1 & 2 & 3 & 4 & 5 & \\
\hline $12: 00$ & 77.71 & 77.70 & 77.69 & 77.71 & 77.69 & $77.7 \pm 0.2$ \\
\hline $12: 04$ & 77.85 & 77.84 & 77.85 & 77.86 & 77.84 & $77.8 \pm 0.2$ \\
\hline $12: 08$ & 77.73 & 77.72 & 77.74 & 77.75 & 77.73 & $77.7 \pm 0.2$ \\
\hline $12: 12$ & 77.86 & 77.85 & 77.86 & 77.84 & 77.85 & $77.9 \pm 0.2$ \\
\hline $12: 16$ & 77.91 & 77.92 & 77.93 & 77.92 & 77.91 & $77.9 \pm 0.2$ \\
\hline $12: 20$ & 77.90 & 77.91 & 77.93 & 77.91 & 77.90 & $77.9 \pm 0.2$ \\
\hline $12: 24$ & 77.85 & 77.84 & 77.83 & 77.86 & 77.85 & $77.8 \pm 0.2$ \\
\hline $12: 28$ & 77.71 & 77.72 & 77.70 & 77.73 & 77.71 & $77.7 \pm 0.2$ \\
\hline $12: 32$ & 77.86 & 77.84 & 77.85 & 77.86 & 77.83 & $77.8 \pm 0.2$ \\
\hline $12: 36$ & 77.87 & 77.85 & 77.84 & 77.86 & 77.85 & $77.9 \pm 0.2$ \\
\hline $12: 40$ & 77.61 & 77.63 & 77.62 & 77.60 & 77.61 & $77.6 \pm 0.2$ \\
\hline $12: 44$ & 77.69 & 77.70 & 77.68 & 77.70 & 77.69 & $77.7 \pm 0.2$ \\
\hline $12: 48$ & 77.61 & 77.59 & 77.62 & 77.61 & 77.59 & $77.6 \pm 0.2$ \\
\hline $12: 52$ & 77.86 & 77.85 & 77.87 & 77.85 & 77.86 & $77.9 \pm 0.2$ \\
\hline $12: 56$ & 77.72 & 77.73 & 77.71 & 77.72 & 77.74 & $77.7 \pm 0.2$ \\
\hline $13: 00$ & 77.83 & 77.85 & 77.84 & 77.83 & 77.84 & $77.8 \pm 0.2$ \\
\hline
\end{tabular}

линии $585 \mathrm{~nm}$ других линий, которые могли бы попасть в полосу пропускания светофильтра, нет.

Такая измерительная схема позволяет обойтись без монохроматора (спектрометра) - прибора достаточно дорогого, а также имеющего значительные габариты, массу и стоимость.

Для достижения высокой стабильности интенсивности излучения ЛСП упомянутый фотодиод в устройстве 4 и элементы 5-7 (рисунок) термостабилизированы. Это определяется тем, что спектральная характеристика фильтра и чувствительность упомянутых фотодиодов имеет заметно выраженную зависимость от температуры окружающей среды. Термостабилизация (с погрешностью поддержания температуры в пределах 2 K) практически полностью исключает влияние изменения температуры окружающей среды на интенсивность излучения ЛСП.

Измерения интенсивности выбранной спектральной линии проводились в течение 4 разделенных промежутками дней, промежуток между первым и последним днем измерений был более месяца. Каждый день цикл измерений составлял от 1 до $3 \mathrm{~h}$. Измерения проводились сериями по 5 измерений с интервалом между сериями в $4 \mathrm{~min}$. Изменения температуры в лабораторном помещении за время каждого из циклов измерений не превышали $3 \mathrm{~K}$.

Обработка результатов проводилась согласно ГОСТ 8.207-76 и [7]. При этом рассчитывались среднее значение силы тока контрольного фотодиода и среднеквадратическое отклонение (СКО) серии с доверительной вероятностью $P=0.95$.

Результаты измерений сведены в табл. 1-4.

Среднее значение силы тока фотодиода $I_{p d}$ по всему массиву измерений от 13.04.2017 г. составляло
Таблица 2. Результаты измерений от 15.04.2017 г.

\begin{tabular}{|c|c|c|c|c|c|c|}
\hline \multirow{2}{*}{$\begin{array}{l}\text { Время, } \\
\text { h:min }\end{array}$} & \multicolumn{5}{|c|}{ Величина тока фотодиода $I_{p d}, \mathrm{nA}$} & \multirow{2}{*}{$\begin{array}{c}\text { Среднее } \\
\text { значение } \\
\text { с СКО }\end{array}$} \\
\hline & 1 & 2 & 3 & 4 & 5 & \\
\hline $14: 40$ & 77.66 & 77.68 & 77.67 & 77.68 & 77.66 & $77.7 \pm 0.2$ \\
\hline $14: 44$ & 77.75 & 77.76 & 77.76 & 77.77 & 77.75 & $77.8 \pm 0.2$ \\
\hline $14: 48$ & 77.80 & 77.79 & 77.80 & 77.81 & 77.80 & $77.8 \pm 0.2$ \\
\hline $14: 52$ & 77.65 & 77.64 & 77.65 & 77.63 & 77.64 & $77.6 \pm 0.2$ \\
\hline $14: 56$ & 77.80 & 77.82 & 77.81 & 77.83 & 77.80 & $77.8 \pm 0.2$ \\
\hline $15: 00$ & 77.79 & 77.80 & 77.79 & 77.78 & 77.80 & $77.8 \pm 0.2$ \\
\hline $15: 04$ & 77.85 & 77.86 & 77.87 & 77.87 & 77.80 & $77.9 \pm 0.2$ \\
\hline $15: 08$ & 77.69 & 77.71 & 77.70 & 77.68 & 77.70 & $77.7 \pm 0.2$ \\
\hline $15: 12$ & 77.79 & 77.81 & 77.80 & 77.82 & 77.80 & $77.8 \pm 0.2$ \\
\hline $15: 16$ & 77.77 & 77.78 & 77.79 & 77.81 & 77.80 & $77.8 \pm 0.2$ \\
\hline $15: 20$ & 77.61 & 77.63 & 77.62 & 77.60 & 77.61 & $77.6 \pm 0.2$ \\
\hline $15: 24$ & 77.75 & 77.76 & 77.74 & 77.76 & 77.75 & $77.8 \pm 0.2$ \\
\hline $15: 28$ & 77.76 & 77.75 & 77.78 & 77.77 & 77.75 & $77.8 \pm 0.2$ \\
\hline $15: 32$ & 77.81 & 77.79 & 77.80 & 77.81 & 77.78 & $77.8 \pm 0.2$ \\
\hline $15: 36$ & 77.81 & 77.82 & 77.80 & 77.79 & 77.80 & $77.8 \pm 0.2$ \\
\hline $15: 40$ & 77.68 & 77.71 & 77.70 & 77.69 & 77.71 & $77.7 \pm 0.2$ \\
\hline
\end{tabular}

Таблица 3. Результаты измерений от 25.04.2017 г.

\begin{tabular}{|c|c|c|c|c|c|c|}
\hline \multirow{2}{*}{$\begin{array}{l}\text { Время, } \\
\text { h:min }\end{array}$} & \multicolumn{5}{|c|}{ Величина тока фотодиода $I_{p d}, \mathrm{nA}$} & \multirow{2}{*}{$\begin{array}{c}\text { Среднее } \\
\text { значение } \\
\text { с СКО }\end{array}$} \\
\hline & 1 & 2 & 3 & 4 & 5 & \\
\hline $12: 00$ & 77.84 & 77.85 & 77.86 & 77.85 & 77.84 & $77.8 \pm 0.2$ \\
\hline $12: 04$ & 77.63 & 77.64 & 77.63 & 77.63 & 77.64 & $77.6 \pm 0.2$ \\
\hline $12: 08$ & 77.69 & 77.68 & 77.69 & 77.68 & 77.68 & $77.7 \pm 0.2$ \\
\hline $12: 12$ & 77.78 & 77.79 & 77.80 & 77.80 & 77.81 & $77.8 \pm 0.2$ \\
\hline $12: 16$ & 77.69 & 77.69 & 77.70 & 77.69 & 77.70 & $77.7 \pm 0.2$ \\
\hline $12: 20$ & 77.64 & 77.63 & 77.64 & 77.63 & 77.64 & $77.6 \pm 0.2$ \\
\hline $12: 24$ & 77.74 & 77.73 & 77.74 & 77.73 & 77.74 & $77.7 \pm 0.2$ \\
\hline $12: 28$ & 77.80 & 77.81 & 77.79 & 77.80 & 77.81 & $77.8 \pm 0.2$ \\
\hline $12: 32$ & 77.69 & 77.70 & 77.69 & 77.70 & 77.70 & $77.7 \pm 0.2$ \\
\hline $12: 36$ & 77.75 & 77.76 & 77.77 & 77.76 & 77.75 & $77.8 \pm 0.2$ \\
\hline $12: 40$ & 77.67 & 77.69 & 77.68 & 77.70 & 77.69 & $77.7 \pm 0.2$ \\
\hline $12: 44$ & 77.76 & 77.75 & 77.75 & 77.74 & 77.75 & $77.8 \pm 0.2$ \\
\hline $12: 48$ & 77.78 & 77.79 & 77.80 & 77.79 & 77.78 & $77.8 \pm 0.2$ \\
\hline $12: 52$ & 77.61 & 77.62 & 77.61 & 77.60 & 77.61 & $77.6 \pm 0.2$ \\
\hline $12: 56$ & 77.78 & 77.77 & 77.78 & 77.76 & 77.75 & $77.8 \pm 0.2$ \\
\hline 13:00 & 77.56 & 77.57 & 77.58 & 77.57 & 77.56 & $77.6 \pm 0.2$ \\
\hline 13:04 & 77.66 & 77.67 & 77.65 & 77.66 & 77.67 & $77.7 \pm 0.2$ \\
\hline 13:08 & 77.69 & 77.70 & 77.71 & 77.72 & 77.71 & $77.7 \pm 0.2$ \\
\hline $13: 12$ & 77.53 & 77.52 & 77.53 & 77.52 & 77.53 & $77.5 \pm 0.2$ \\
\hline $13: 16$ & 77.50 & 77.49 & 77.51 & 77.50 & 77.50 & $77.5 \pm 0.2$ \\
\hline $13: 20$ & 77.71 & 77.70 & 77.69 & 77.70 & 77.69 & $77.7 \pm 0.2$ \\
\hline $13: 24$ & 77.75 & 77.74 & 77.73 & 77.74 & 77.75 & $77.7 \pm 0.2$ \\
\hline
\end{tabular}

$77.76 \pm 0.17 \mathrm{nA}$, по массиву измерений от 15.04.2017 г. составляло $77.74 \pm 0.14 \mathrm{nA}$, по массиву измерений от 25.04 .2017 г. составляло $77.68 \pm 0.19 \mathrm{nA}$, по массиву измерений от 16.05.2017 г. составляло $77.60 \pm 0.22 \mathrm{nA}$. Полученные данные говорят о том, что сила тока 
Таблица 4. Результаты измерений от 16.05.2017 г.

\begin{tabular}{|c|c|c|c|c|c|c|}
\hline \multirow{2}{*}{$\begin{array}{c}\text { Время, } \\
\text { h:min }\end{array}$} & \multicolumn{5}{|c|}{ Величина тока фотодиода $I_{p d}, \mathrm{nA}$} & \multirow{2}{*}{$\begin{array}{c}\text { Среднее } \\
\text { значение } \\
\text { с СКО }\end{array}$} \\
\hline & 1 & 2 & 3 & 4 & 5 & \\
\hline 13:00 & 77.79 & 77.81 & 77.78 & 77.79 & 77.78 & $77.8 \pm 0.2$ \\
\hline 13:04 & 77.78 & 77.77 & 77.77 & 77.76 & & $77.8 \pm 0.2$ \\
\hline 13:08 & 77.71 & 77.69 & 77.72 & 77.70 & 77.71 & \\
\hline $13: 12$ & 77.68 & 77.69 & 77.70 & 77.69 & 77.70 & \\
\hline 13:16 & 77.76 & 77.75 & 77.75 & 77.76 & 77.75 & $77.8 \pm 0.2$ \\
\hline $13: 20$ & 77.67 & 77.64 & 77.65 & 77.65 & 77.67 & $77.7 \pm 0.2$ \\
\hline $13: 24$ & 77.80 & 77.82 & 77.80 & 77.81 & 77.81 & $77.8 \pm 0.2$ \\
\hline $13: 28$ & 77.76 & 77.78 & & & & \\
\hline $13: 32$ & 77.77 & 77. & 77.77 & 77.75 & 77.78 & \\
\hline $13: 36$ & 77.68 & 77.66 & 77.65 & 77.64 & 77.64 & \\
\hline $13: 40$ & 77.82 & 77.81 & 77.79 & 77.79 & 77.78 & $77.8 \pm 0.2$ \\
\hline $13: 44$ & 77.62 & 77. & 77.62 & 77.63 & 77.62 & \\
\hline $13: 48$ & 77.66 & 77. & 77. & 77.6 & 77. & \\
\hline $13: 52$ & 77.66 & 77. & 77.66 & 77. & 77. & \\
\hline $13: 56$ & 77.81 & 77. & 77. & 77. & 77.80 & \\
\hline $14: 00$ & 77.65 & 77. & 77.67 & 77.6 & 77.65 & $77.7 \pm 0.2$ \\
\hline 14:04 & .77 & & & & & \\
\hline 14: & .63 & & & & & \\
\hline $14: 12$ & 77.69 & & 77. & & 77 & \\
\hline 14:16 & 77.61 & 77.60 & 77.59 & 77.59 & 77.61 & $77.6 \pm 0.2$ \\
\hline $14: 20$ & 7.69 & 77. & 77. & 66 & 77. & \\
\hline $14: 24$ & 77.62 & 77.63 & 77.61 & 77. & 77.60 & \\
\hline $14: 28$ & 77.67 & 77. & 77.6 & 77.69 & 77. & \\
\hline $14: 32$ & 77.70 & & & 77. & 77.70 & \\
\hline $14: 36$ & 77.69 & 77. & 77. & 77. & 77.70 & \\
\hline $14: 40$ & 77.68 & 77. & 77.70 & 77. & 77.69 & \\
\hline $14: 44$ & 77.60 & & & & & \\
\hline $14: 48$ & 77.76 & & & & 77. & \\
\hline $14: 52$ & 77.78 & 77.77 & 77.78 & 77.79 & 77.77 & $77.8 \pm 0.2$ \\
\hline $14: 56$ & 77.67 & 77.6 & 77.67 & 77.67 & 77.66 & \\
\hline $15: 00$ & 77.74 & 77.75 & 77.76 & 77.76 & 77.77 & \\
\hline $15: 04$ & 77.75 & & 77.72 & & & $77.7 \pm 0.2$ \\
\hline $15: 08$ & 77.72 & 77.70 & 77.71 & 77.73 & 77.70 & $77.7 \pm 0.2$ \\
\hline $15: 12$ & 77.77 & 77. & 77.73 & 77.75 & 77.76 & $77.8 \pm 0.2$ \\
\hline $15: 16$ & 77.61 & 77.60 & 77.58 & 77.57 & 77.59 & 0.2 \\
\hline $15: 20$ & 77.56 & 77.58 & 77.55 & 77.54 & 77.56 & $77.6 \pm 0.2$ \\
\hline $15: 24$ & 77.56 & 77.5 & 77.56 & 77.57 & 77.55 & $77.6 \pm 0.2$ \\
\hline & 77.42 & 77.43 & 77.45 & 77.45 & 77.43 & $77.4 \pm 0.2$ \\
\hline $15: 32$ & 77.49 & 77.46 & 77.50 & 77.47 & 77.51 & $77.5 \pm$ \\
\hline & & & 77.51 & 77.49 & 77.51 & $77.5 \pm 0.2$ \\
\hline $15: 40$ & 77.60 & 77.58 & 77.61 & 77.59 & 77.58 & $77.6 \pm 0.2$ \\
\hline $15: 44$ & 77.43 & 77.41 & 77.40 & 77.38 & 77.41 & $77.4 \pm 0.2$ \\
\hline & & & 77.47 & 77.45 & 77.44 & \\
\hline $15: 52$ & 77.41 & 77.42 & 77.43 & 77.42 & 77.40 & $77.4 \pm 0.2$ \\
\hline $15: 56$ & 77.49 & 77.50 & 77.50 & 77.51 & 77.53 & $77.5 \pm 0.2$ \\
\hline $16: 00$ & 77.70 & 77.67 & 77.72 & 77.68 & 77.70 & $77.7 \pm 0.2$ \\
\hline
\end{tabular}

контрольного фотодиода при работе ЛСП неизменна с погрешностью менее $0.2 \%$.

Поскольку в выбранном диапазоне токов зависимость силы тока фотодиода от освещенности линейна [8], то можно сделать вывод, что интенсивность анализируемой спектральной линии как в кратковременном интервале (несколько минут), так и в долговременном (более месяца) изменяется менее чем на $0.2 \%$.

Надо отметить, что рассмотренный высокостабильный источник спектральных линий является прототипом средств измерений (СИ) для метрологии оптико-физических измерений. Поэтому параметры окружающей среды, при которых были выполнены представленные результаты, соответствуют типовым режимам стационарных лабораторий. Учитывая, что измерения выполнены весной 2017 г., можно указать диапазон изменения температуры окружающей среды (за время измерений) $+21 \ldots+23^{\circ} \mathrm{C}$. Влажность воздуха и атмосферное давление не контролировались.

Это объясняется тем, что для разработанного высокостабильного источника спектральных линий принципиально важна долговременная стабильность. Существенное влияние на нее оказывает „старение“ полупроводниковых элементов. Отмеченный факт является практически единственной причиной нестабильности метрологических характеристик СИ оптико-физических измерений.

На базе предложенного высокостабильного источника спектральных линий готовится к прохождению государственных испытаний СИ, предназначенное для спектроскопических измерений с соответствующей метрологической аттестацией.

\section{Выводы}

В работе описан разработанный и изготовленный высокостабильный источник линейчатого спектра на основе лампы с полым катодом. Для достижения требуемой стабильности интенсивности излучаемых спектральных линий в контур управления ЛСП введена отрицательная обратная связь, сигнал которой формируется из выходного светового сигнала ЛСП фотодиодным датчиком с усилителем-преобразователем. Подобное техническое решение позволило получить высокий уровень стабильности спектральных линий - интенсивность анализируемой спектральной линии как в кратковременном интервале (несколько минут), так и в долговременном (более месяца) изменяется менее чем на $0.2 \%$.

Предоставленные в статье результаты исследований получены в рамках выполнения государственного задания Минобрнауки России № 8.5577.2017/8.9 на выполнение проекта по теме „Исследование шумовых характеристик и пульсаций микросхем мобильных источников вторичного электропитания“.

\section{Список литературы}

[1] Львов Б.В. Атомно-абсорбционный спектральный анализ. М.: Наука, 1966. 392 с.

[2] Хавезов И. Атомно-абсорбционный анализ. Пер. с болг. / Под ред. С.З. Яковлевой. Л.: Химия, 1983. 144 с. 
[3] Барун В.В., Иванов А.П. // Опт. и спектр. 2009. Т. 106. № 1. C. 89; Barun V.V., Ivanov A.P. // Opt. Spectrosc. 2009. V. 106. N 1. P. 84.

[4] Захаров Ю.А., Кокорина О.Б., Окунев Р.В. // Опт. и спектр. 2014. T. 116. № 4. C. 692; Zakharov Yu.A., Kokorina O.B., Okunev R.V. // Opt. Spectrosc. 2014. V. 116. N 4. P. 642.

[5] Битюков В.К., Симачков Д.С. Источники вторичного электропитания. М.: Инфра-Инженерия, 2017. 326 с.

[6] Битюков В.К., Иванов А.А., Миронов А.В., Михневич Н.Г, Перфильев В.С., Петров В.А. // Российский технологический журнал. 2016. Т. 4. № 3 (12). С. 37.

[7] Сигов А.С., Белик Ю.Д., Верба В.С., Нефедов В.И., Битюков В.К., Хахин В.И. Метрология и электрорадиоизмерения в телекоммуникационных системах. Изд. 2-е, перераб. и доп. М.: Высшая школа, 2005. 536 с.

[8] Шило В.Л. Линейные интегральные схемы в радиоэлектронной аппаратуре. М.: Советское радио, 1979. 368 с. 